\title{
Design of a plant of obtaining de biodiesel to leave of a residual of the sugar industry
} (phlegm)

\author{
Dr. Ing. Juan B. de León Benítez ${ }^{1}$ Lesdier Abreu Rodríguez ${ }^{2}$ \\ ${ }^{1,2}$ Universidad Central "Marta Abreu” de Las Villas, Facultad de Química-Farmacia. Carretera a Camajuaní, Km 5 1/2, Santa \\ Clara, Villa Clara, Cuba. CP 50100
}

\begin{abstract}
In this paper an analysis of the investment task and projection as well as technical analysis is performed. Economic possibilities Biodiesel production on an industrial scale in the honey "Heriberto Duquesne" Company. An extensive literature search on the subject of biofuels and biodiesel in particular with a view to selecting the most feasible variant of production and raw materials was conducted to carry out the process, where the oil filter cake, ethyl alcohol and sodium ethoxide catalyst were the most attractive variants. The essential part of the job is the task of investment and projection, followed by sizing equipment for the production of Biodiesel plant, from the residual filter cake as providing the Company honeypot. Additionally material balances and energy are analyzed, in addition to equipment design of the chosen section, as wellas their technical - economical analysis.
\end{abstract}

Key words: biodiesel, feasible, oil, phlegm, ethylic alcohol, etóxido.

\section{Diseño de una planta de obtención de biodiesel a partir de un residual de la industria azucarera (cachaza)}

\section{RESUMEN}

En el presente trabajo se realiza un análisis de la tarea de inversión y de proyección, así como un análisis técnico - económico de las posibilidades de producción de Biodiesel a escala industrial en la Empresa Mielera "Heriberto Duquesne". Se realizó una extensa búsqueda bibliográfica sobre el tema de los biocombustibles y en particular del biodiesel con vistas a seleccionar la variante de producción más factible, así como las materias primas para llevar a cabo el proceso, donde el aceite de cachaza, el alcohol etílico y el catalizador etóxido de sodio fueron las variantes más atractivas. La parte esencial del trabajo consiste con la tarea de inversión y de proyección, seguida del dimensionamiento de los equipos para la planta de obtención de Biodiesel, a partir de la cachaza residual que aporta dicha Empresa Mielera. Además se analizan los balances de materiales y los de energía, además del diseño de los equipos de la sección escogida, así como su análisis técnico - económico.

Palabras clave: biodiesel, factible, aceite, cachaza, alcohol etílico, etóxido.

\section{INTRODUCCIÓN}

La búsqueda de combustibles alternativos que mitiguen el efecto negativo de los combustibles fósiles en el medio ambiente y en la economía mundial es una necesidad de la humanidad hasta tanto no se desarrolle una forma radical de sustituir la matriz energética actual basada en la combustión de estos últimos. La utilización de los biocombustibles no es una solución definitiva al problema de la energía, solo se utilizaría para la transición a otros tipos de tecnologías más avanzadas y baratas, dentro de los biocombustibles [1].
La búsqueda de fuentes y tecnologías alternativas de energía debe ser una de las prioridades mundiales en los próximos años y décadas. Dos características de estas nuevas fuentes y tecnologías son esenciales: la minimización del impacto ambiental y la renovabilidad. Una de los problemas más frecuentes en éste campo es la adaptabilidad de dichas energías a la vida cotidiana, ya que la transición hacia éstas acarrea costos frecuentemente insuperables. Sin embargo, el desarrollo de los llamados biocombustibles, se presenta como una alternativa alentadora, siempre y cuando no obtengan a partir de semillas o plantas que puedan servir de alimentos, ni comprometan los cultivos de las tierras destinadas a estos [2]. 
Juan B. de León Benítez \& Lesdier Abreu Rodríguez/ITEGAM-JETIA Vol.01, № 04, pp.19-24. Dezembro, 2015.

El biodiesel es un producto que ha tomado relevancia a nivel internacional a partir de la crisis experimentada por los combustibles fósiles, producto de su escasez y de los problemas ambientales que traen consigo. Se han estudiado muchas fuentes para su obtención, la mayoría de las cuales atentan de una forma u otra al desarrollo alimenticio de los países pobres que dependen de la agricultura y además poseen un elevado costo para su obtención; las materias primas de bajo costo (como los aceites usados o grasas animales), requieren complejos procesos de purificación para disminuir el contenido de humedad y de acidez, lo que hace que el proceso sea muchas veces no rentable. Sin embargo la cera de caña que es una fuente de ácidos grasos no ha sido objeto de atención a nivel mundial.

La utilización de la cera como fuente para la producción de biodiesel es una forma de obtener un biocombustible sin utilizar como materia prima fuentes de alimentos, ni emplear tierras para el cultivo de materias primas con este fin. El Biodiesel se produce a partir de los aceites vegetales u animales convirtiendo estos a ésteres de etilo, a través de un proceso denominado transesterificación, usando etanol o metanol en presencia de un catalizador básico. La reacción se lleva a cabo entre 25 y $80^{\circ} \mathrm{C}$, con agitación mecánica. Además del biodiesel (etil ésteres), en la reacción de transesterificación, se obtiene un producto de un alto valor agregado: la glicerina o los alcoholes de alto peso molecular (AAPM), de acuerdo a la materia prima empleada; estos pueden ser comercializados, ayudando a la factibilidad económica de la planta [3][4].

\section{DESCRIPCIÓN DEL PROCESO}

Extracción del aceite de la cachaza: esta primera etapa tiene como propósito extraer el aceite de la cera de la cachaza, utilizando como solvente orgánico la nafta; la proporción utilizada es de 3 Litros por $\mathrm{Kg}$ de cachaza. La operación se realiza en un mezclador por una hora a una temperatura de $65{ }^{\circ} \mathrm{C}$ [5].
Recuperación del solvente orgánico: el solvente se recupera mediante la evaporación, logrando incorporarla al proceso hasta un $97 \%$ del mismo. En esta etapa además se obtiene el aceite de cachaza "limpio", el cual es posteriormente utilizado en la obtención de biodiesel; por tanto, la calidad de esta operación garantiza la calidad del producto final [6].

Deshidratación de etanol: esta etapa tiene como objetivo obtener el alcohol deshidratado (99 $\left.{ }^{0} \mathrm{GL}\right)$ necesario para la producción de biodiesel, el cual se logra en una columna de adsorción de cama fija utilizando como absolvente la zeolita natural. Esta etapa es decisiva para lograr que se forme Biodiesel y no otros productos que pueden surgir en caso de que ocurra la saponificación si el alcohol presenta alguna cantidad de agua [7].

Preparación del etóxido: no es más que preparar una solución de etanol obtenido en la etapa anterior con Hidróxido de sodio, el cual es el agente catalizador de la próxima etapa. La solución se prepara a una temperatura de $65^{\circ} \mathrm{C}$.

Etapa de reacción (transesterificación): en esta etapa ocurre la reacción de transesterificación, donde se hace reaccionar al aceite libre de solvente y el etóxido; de la misma se obtiene el Biodiesel y los Alcoholes de Alto Peso Molecular, los cuales pueden ser aprovechados por otros sectores industriales. Esta mezcla fuertemente cáustica es vertida en el reactor principal que contiene los líquidos fundidos. La reacción se realizará a $65{ }^{\circ} \mathrm{C}$ para agilizar la misma y se mezcla vigorosamente para favorecer una reacción completa. Después de transcurridos 50 minutos, se procede a una separación de los coproductos (los alcoholes pesados); luego de separar la misma, el éter es lavado para eliminar jabones y otros subproductos de reacción indeseados, los cuales pueden contaminar el producto principal [9].

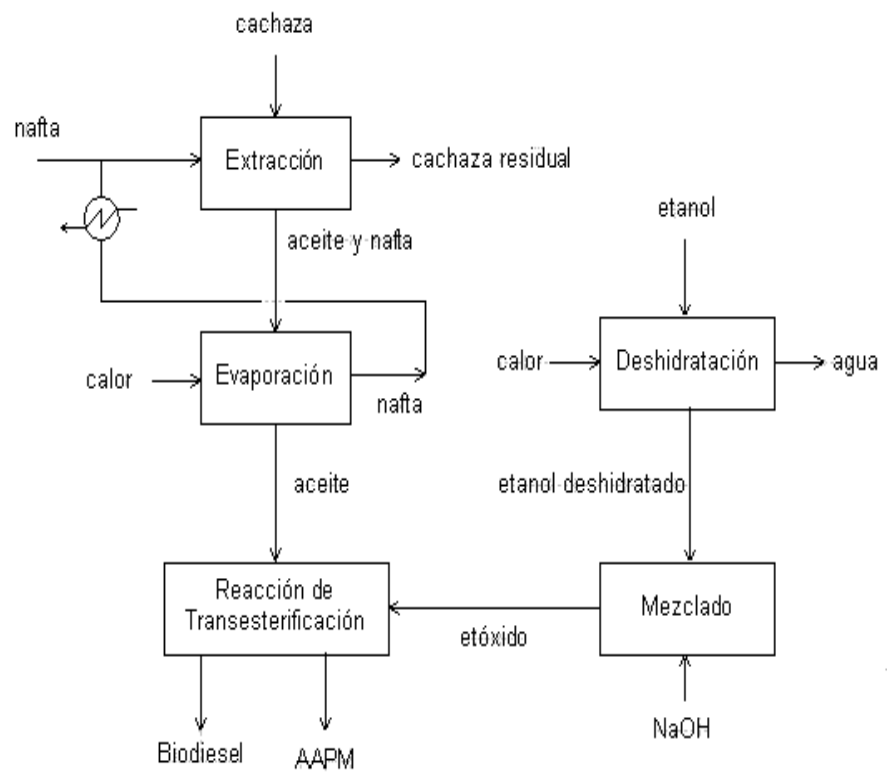

Figura.1. Diagrama del proceso de obtención de Biodiesel a partir de la cachaza [4]. 
Juan B. de León Benítez \& Lesdier Abreu Rodríguez/ITEGAM-JETIA Vol.01, No 04, pp.19-24. Dezembro, 2015.

Balances de masa y de energía de los equipos seleccionados.

Tabla 1 - Extracción del aceite de la cachaza. (Base de cálculo 1día)

\begin{tabular}{|c|c|c|}
\hline Datos & Fórmulas & Resultados \\
\hline \multicolumn{3}{|c|}{ Balance de masa } \\
\hline$\%$ humedad $=13$ & $\mathrm{~F}=\%$ aceite en cachaza $*(\mathrm{~m}$ cachaza $-\mathrm{m}$ cachaza $* \%$ humedad $)$ & $\mathrm{F}=2190 \mathrm{Kg}$ \\
\hline$\%$ aceite en cachaza $=12$ (base seca) & $\mathrm{B}=\mathrm{m}_{\text {cachaza }}-\mathrm{F}$ & $\mathrm{B}=18810 \mathrm{Kg}$ \\
\hline$\%$ de extracción $=96,05$ & $\mathrm{~N}_{\mathrm{f}}=\mathrm{B} / \mathrm{F}$ & $\mathrm{N}_{\mathrm{f}}=8,59$ \\
\hline$\%$ pérdida de nafta $=8,69$ & $\mathrm{E}_{1}=\mathrm{Kg}$ aceite $+\mathrm{Kg}$ nafta (en el sólido) & $\mathrm{E}_{1}=5580 \mathrm{Kg}$ \\
\hline $\mathrm{m}_{\text {cachaza }}=21000 \mathrm{Kg}$ & $\mathrm{R}_{1}=\mathrm{Kg}$ aceite $+\mathrm{Kg}$ nafta (en el líquido) & $\mathrm{R}_{1}=59610 \mathrm{Kg}$ \\
\hline $\mathrm{m}_{\text {nafta }}=63000 \mathrm{Kg}$ & $\mathrm{N}_{1}=\mathrm{B} / \mathrm{E}_{1}$ & $\mathrm{~N}_{1}=3,37$ \\
\hline$\rho_{\text {cachaza }}=141.42 \mathrm{Kg} / \mathrm{m}^{3}$ & $\mathrm{X}_{1}=\mathrm{C} /(\mathrm{A}+\mathrm{C})$ en el líquido & $\mathrm{X}_{1}=0,033$ \\
\hline$\rho_{\text {nafta }}=750 \mathrm{Kg} / \mathrm{m}^{3}$ & $\mathrm{Y}_{1}=\mathrm{C} /(\mathrm{A}+\mathrm{C})$ en el sólido & $\mathrm{Y}_{1}=0,0395$ \\
\hline$\rho_{\text {aceite }}=980 \mathrm{Kg} / \mathrm{m}^{3}$ & Aceite en el líquido lixiviado. $=\mathrm{R}_{1} * \mathrm{X}_{1}$ & $\mathrm{R}_{1} * \mathrm{X}_{1}=1967,13 \mathrm{Kg}$ \\
\hline$\rho_{\text {nafta }+ \text { aceite }}=750 \mathrm{Kg} / \mathrm{m}^{3}$ & Aceite en el sólido lixiviado. $=\mathrm{E}_{1} * \mathrm{Y}_{1}$ & $\mathrm{E}_{1} * \mathrm{Y}_{1}=110 \mathrm{Kg}$ \\
\hline & Nafta en el líquido lixiviado. $\mathrm{N}_{\mathrm{L}}=0,9131 * \mathrm{R}_{0}$ & $\mathrm{~N}_{\mathrm{L}}=57525,3 \mathrm{Kg}$ \\
\hline & Nafta en el sólido lixiviado. $\mathrm{N}_{s}=0,0869 * \mathrm{R}_{0}$ & $\mathrm{~N}_{\mathrm{s}}=5474,7 \mathrm{Kg}$ \\
\hline
\end{tabular}

\begin{tabular}{|c|c|c|}
\hline Datos & Fórmulas & Resultados \\
\hline \multicolumn{3}{|c|}{ Balance de energía } \\
\hline $\begin{array}{l}\mathrm{m}_{\text {cachaza }}=2330 \mathrm{Kg} \text { (en cada tanque) } \\
\mathrm{m}_{\text {nafta }}=7000 \mathrm{Kg} \\
\mathrm{Cp}_{\text {cachaza }}=1.145 \mathrm{Kcal} / \mathrm{Kg}^{0} \mathrm{C} \\
\mathrm{Cp}_{\text {nafta }}=0.5 \mathrm{Kcal} / \mathrm{Kg}^{0} \mathrm{C} \\
8 \text { horas de trabajo } \\
\text { Tiempo de duración : } 1 \text { hora } \\
\mathrm{T}_{2}=75^{\circ} \mathrm{C} \\
\mathrm{T}_{1}=25^{\circ} \mathrm{C} \\
\mathrm{T}_{\text {amb }}=28^{0} \mathrm{C} \\
\mathrm{Ps}_{\text {vapor }}=10.68 \mathrm{~atm} \\
\mathrm{Ts}_{\text {vapor }}=183.28^{0} \mathrm{C} \\
\lambda \mathrm{s}=478.63 \mathrm{Kcal} / \mathrm{Kg} \\
\eta=100 \% \text { (eficiencia de la transferencia } \\
\text { de calor). }\end{array}$ & 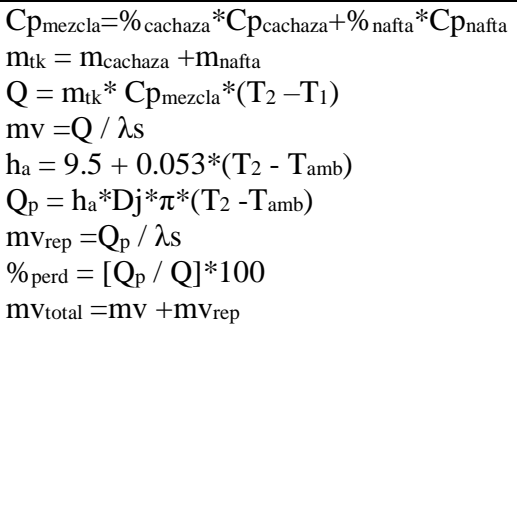 & $\begin{array}{l}\text { Cpmezcla }=0.72 \\
\mathrm{Kcal} / \mathrm{Kg}^{0} \mathrm{C} \\
\mathrm{m}_{\text {tk }}=9330 \mathrm{Kg} \\
\mathrm{Q}=335880 \mathrm{Kcal} \\
\mathrm{mv}=701.75 \mathrm{Kg} \\
\mathrm{h}_{\mathrm{a}}=10.31 \mathrm{Kcal} / \mathrm{hm}^{2} \\
{ }^{0} \mathrm{C} \\
\mathrm{Q}_{\mathrm{p}}=4567.15 \mathrm{Kcal} \\
\mathrm{mv}_{\text {rep }}=9.54 \mathrm{Kg} \\
\%_{\text {perd }}=1.35 \% \\
\mathrm{mv}_{\text {total }}=711.29 \mathrm{Kg}\end{array}$ \\
\hline
\end{tabular}

Tabla 2 - Formulas y Separación de la nafta por evaporación. (Base de cálculo 1 día).

\begin{tabular}{|c|c|c|}
\hline Datos & Fórmulas & Resultados \\
\hline \multicolumn{3}{|c|}{ Balance de masa } \\
\hline $\begin{array}{l}\mathrm{m}_{\text {nafta }} \text { aceite }=59610 \mathrm{Kg} \\
\text { Grado de separación }=100 \% \\
\% \text { aceite } \text { mezcla }=3.3 \%\end{array}$ & 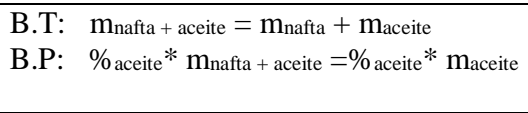 & $\begin{array}{l}\mathrm{m}_{\text {nafta }}=58790 \mathrm{Kg} \\
\mathrm{m}_{\text {aceite }}=817 \mathrm{Kg}\end{array}$ \\
\hline
\end{tabular}

\begin{tabular}{|c|c|c|}
\hline Datos & Fórmulas & Resultados \\
\hline \multicolumn{3}{|c|}{ Balance de energía } \\
\hline $\begin{array}{l}\mathrm{m}_{\text {mezcla }}=59610 \mathrm{Kg} \\
\mathrm{Cp}_{\text {cachaza }}=1.145 \mathrm{Kcal} / \mathrm{Kg}^{0} \mathrm{C} \\
\mathrm{C} p_{\text {nafta }}=0.5 \mathrm{Kcal} / \mathrm{Kg}^{0} \mathrm{C} \\
8 \text { horas de trabajo } \\
\mathrm{T}_{2}=178^{0} \mathrm{C} \\
\mathrm{T}_{1}=40^{\circ} \mathrm{C} \\
\mathrm{T}_{\text {amb }}=28^{0} \mathrm{C} \\
\mathrm{Ps}_{\text {vapor }}=10.68 \mathrm{~atm} \\
\mathrm{~T}_{\text {sapor }}=183.28^{0} \mathrm{C} \\
\lambda \mathrm{s}=478.63 \mathrm{Kcal} / \mathrm{Kg} \\
\text { Pérdidas de nafta }=0.4 \% \\
\eta=100 \%\end{array}$ & $\begin{array}{l}\mathrm{Cp}_{\text {mezcla }}=\%_{\text {aceite }} * C \mathrm{C}_{\text {aceite }}+\%_{\text {nafta }} * \mathrm{Cp}_{\text {nafta }} \\
\mathrm{mv}=\left[(\mathrm{m} * \mathrm{Cp})_{\text {mezcla }} *\left(\mathrm{~T}_{2}-\mathrm{T}_{1}\right)+(\mathrm{m} * \lambda)_{\text {nafta }}\right] / \lambda \mathrm{s} \\
\mathrm{Q}=\mathrm{mv} * \lambda \mathrm{s} \\
\mathrm{h}_{\mathrm{a}}=9.5+0.053 *\left(\mathrm{~T}_{2}-\mathrm{T}_{\mathrm{amb}}\right) \\
\mathrm{Q}_{\mathrm{p}}=0.1 * \mathrm{Q} \\
\mathrm{m} v_{\text {rep }}=\mathrm{Q}_{\mathrm{p}} / \lambda \mathrm{s} \\
\%_{\text {perd }}=\left[\mathrm{Q}_{\mathrm{p}} / \mathrm{Q}\right]^{*} 100 \\
\mathrm{mv} \text { total }=\mathrm{mv}+\mathrm{mv}_{\text {rep }}\end{array}$ & $\begin{array}{l}\mathrm{Cp}_{\text {mezcla }}=0.52 \mathrm{Kcal} / \mathrm{Kg}^{0} \mathrm{C} \\
\mathrm{mv}=18126.28 \mathrm{Kg} \\
\mathrm{Q}=8675782.1 \mathrm{Kcal} \\
\mathrm{h}_{\mathrm{a}}=15.004 \mathrm{Kcal} / \mathrm{hm}^{2}{ }^{0} \mathrm{C} \\
\mathrm{Q}_{\mathrm{p}}=867578.21 \mathrm{Kcal} \\
\mathrm{mv} \text { rep }=1812.63 \mathrm{Kg} \\
\%_{\text {perd }}=10 \% \\
\mathrm{mv} \text { total }=19938.91 \mathrm{Kg}\end{array}$ \\
\hline
\end{tabular}


Juan B. de León Benítez \& Lesdier Abreu Rodríguez/ITEGAM-JETIA Vol.01, No 04, pp.19-24. Dezembro, 2015.

Separación de la nafta por evaporación. (Base de cálculo 1 día).

Tabla 3 - Condensación de la nafta. (Base de cálculo 1 día).

No presenta balances de masa ya que es un intercambiador de calor tubo y concha donde lo que entra por cada fluido es lo que sale em ellos mismos.

\begin{tabular}{|c|c|c|}
\hline Datos & Fórmulas & Resultados \\
\hline \multicolumn{3}{|c|}{ Balance de energía } \\
\hline $\begin{array}{l}\mathrm{m}_{\text {nafta }}=58790 \mathrm{Kg} \\
\lambda_{\text {nafta }}=76.45 \mathrm{Kcal} / \mathrm{Kg} \\
\mathrm{C}_{\text {agua }}=1 \mathrm{Kcal} / \mathrm{Kg}^{0} \mathrm{C} \\
\mathrm{T}_{2} \text { agua } \\
\mathrm{T}_{1} \text { agua } \\
=20^{\circ} \mathrm{C} \\
\mathrm{T}_{\text {nafta }}=178^{\circ} \mathrm{C} \\
8 \text { horas de trabajo } \\
\eta=100 \%\end{array}$ & $\begin{array}{l}\mathrm{m}_{\text {agua }}=\mathrm{m}_{\text {nafta }} * \lambda_{\text {nafta }} /\left[\mathrm{Cp}_{\text {agua }} *\left(\mathrm{~T}_{2}-\mathrm{T}_{1}\right)\right] \\
\mathrm{m}_{\text {agua }}=\mathrm{m}_{\text {agua }} / 8 \\
\mathrm{Q}=\mathrm{m}_{\text {nafta }} * \lambda_{\text {nafta }}\end{array}$ & $\begin{array}{l}\mathrm{m}_{\text {agua }}=299633.03 \mathrm{Kg} \\
\mathrm{m}_{\text {agua }}=37454.13 \mathrm{Kg} / \mathrm{h} \\
\mathrm{Q}=4494495.5 \mathrm{Kcal}\end{array}$ \\
\hline
\end{tabular}

Tabla 4 - Diseño del tanque de mezclado de la cachaza en la nafta.

Para esta etapa donde se extraerá el aceite, se procesarán 21 ton/d de cachaza. Según las experiencias realizadas al respecto, la cantidad de solvente (nafta) es $3 \mathrm{~L} / \mathrm{Kg}$ de cachaza alimentada.

\begin{tabular}{|l|l|l|}
\hline \multicolumn{1}{|c|}{ Datos } & \multicolumn{1}{|c|}{ Fórmulas } & \multicolumn{1}{c|}{ Resultados } \\
\hline $\mathrm{m}_{\text {cachaza }}=2330 \mathrm{Kg}$ & $\mathrm{m}_{\text {mezcla }}=\mathrm{m}_{\text {cachaza }}+\mathrm{m}_{\text {nafta }}$ & $\mathrm{m}_{\text {mezcla }}=9330 \mathrm{Kg}$ \\
$\mathrm{m}_{\text {nafta }}=7000 \mathrm{Kg}$ & $\rho_{\text {mezcla }}=\sum \mathrm{x}_{\mathrm{i}}{ }^{\circ} \rho_{\mathrm{i}}$ & $\rho_{\text {mezcla }}=549.17 \mathrm{Kg} / \mathrm{m}^{3}$ \\
$\rho_{\text {cachaza }}=141.42 \mathrm{Kg} / \mathrm{m}^{3}$ & $\mathrm{~V}=\mathrm{m}_{\text {mezcla }} / \rho_{\text {mezcla }}$ & $\mathrm{V}=16.99 \mathrm{~m}^{3}$ \\
$\rho_{\text {nafta }}=750 \mathrm{Kg} / \mathrm{m}^{3}$ & $\mathrm{~V}_{\text {sd }}=25 \% * \mathrm{~V}+\mathrm{V}$ & $\mathrm{V}_{\mathrm{sd}}=21.24 \mathrm{~m}^{3}$ \\
$25 \%$ de sobrediseño & $\mathrm{D}=\left[(4 * \mathrm{~V}) /\left(1.5^{*} \pi\right)\right]^{1 / 3}$ & $\mathrm{D}=2.62 \mathrm{~m}$ \\
$\mathrm{H} / \mathrm{D}=1.5 \stackrel{\mathrm{H}=1.5 * \mathrm{D}}{\mathrm{T}}$ & $\mathrm{H}=1.5 * \mathrm{D}$ & $\mathrm{H}=3.93 \mathrm{~m}$ \\
Tanque cilíndrico & & \\
\hline
\end{tabular}

Tabla 5 - Diseño de un agitador de hélice.

El agitador de hélice se escoge, porque cumple con todos los requerimientos de esta etapa, es decir, provocar la agitación en grandes volúmenes de líquidos, bajo costo, bajo consumo de energía y además se pueden colocar varios agitadores en el mismo eje para asegurar el mezclado en todo el recipiente.

\begin{tabular}{|c|c|c|c|}
\hline Datos & Fórmulas & Resultados & Referencia \\
\hline $\begin{array}{l}D_{\text {tanque }}=2.62 \mathrm{~m} \\
\mathrm{H}_{\text {tanque }}=3.93 \mathrm{~m} \\
\mathrm{n}=180 \mathrm{rpm}=3 \mathrm{rps} \\
\rho_{\text {mezcla }}=549.17 \mathrm{Kg} / \mathrm{m}^{3} \\
\mu_{\text {mezcla }}=0.1 \mathrm{~Pa} . \mathrm{s} \\
\mathrm{H} / \mathrm{D}=1.5 \\
\text { Curva N } 6^{(1)}\end{array}$ & $\begin{array}{l}\mathrm{d}=0.3 * \mathrm{D}^{(1)} \\
\operatorname{Re}_{\text {mez }}=\left[\mathrm{n}^{*}\left(\mathrm{D}^{2}\right)^{*} \rho\right] \\
/ \mu^{(2)} \\
\mathrm{Kn}_{\text {correg }}=\mathrm{Kn} *(\mathrm{H} / \\
\mathrm{D})^{0.5(2)} \\
\mathrm{N} \\
\mathrm{Kn}_{\text {correg }} * \rho^{*}\left(\mathrm{n}^{3}\right)^{*}\left(\mathrm{D}^{5}\right) \\
(2) \\
\mathrm{N}_{\text {arranque }}=2 * \mathrm{~N}^{(2)}\end{array}$ & $\begin{array}{l}\mathrm{d}=0.79 \mathrm{~m} \\
\text { (diámetro del agitador) } \\
\text { Re }_{\text {mez }}=1.13^{*} 10^{5} \\
\text { (régimen turbulento) } \\
\mathrm{Kn}=0.18^{(4)} \\
\mathrm{Kn}_{\text {correg }}=0.22 \\
\mathrm{~N}=402.72 \mathrm{KW} \\
\mathrm{N}_{\text {arranque }}=805.44 \mathrm{KW}\end{array}$ & $\begin{array}{l}\text { (1) Fig. 21 Apéndice } \\
\text { (2) Rosabal, J } \\
{ }^{(3)} \text { Tabla } 9.1 \\
\text { (4) Fig. } 9.7\end{array}$ \\
\hline
\end{tabular}

Tabla 6 - Diseño del tanque de evaporación de la nafta.

La capacidad del tanque evaporador se determinará para procesar toda la mezcla de aceite extraído y nafta utilizada como solvente en el tanque de extracción. La mezcla de aceite + nafta que se obtiene en el tanque extractor es $59610 \mathrm{Kg} / \mathrm{d}$.

\begin{tabular}{|c|c|c|}
\hline Datos & Fórmulas & Resultados \\
\hline $\begin{array}{l}\mathrm{m}_{\text {mezcla }}=9330 \mathrm{Kg} \\
\rho_{\text {mezcla }}=549.17 \mathrm{Kg} / \mathrm{m}^{3} \\
25 \% \text { de sobrediseño } \\
\mathrm{H} / \mathrm{D}=1.5 \underset{\mathrm{H}}{\longrightarrow}=1.5 * \mathrm{D} \\
\text { Tanque cilíndrico }\end{array}$ & $\begin{array}{l}\mathrm{V}=\mathrm{m}_{\text {mezcla }} / \rho_{\text {mezcla }} \\
\mathrm{V}_{\text {sd }}=25 \% * \mathrm{~V}+\mathrm{V} \\
\mathrm{D}=[(4 * \mathrm{~V}) /(1.5 * \pi)]^{1 / 3} \\
\mathrm{H}=1.5 * \mathrm{D}\end{array}$ & $\begin{array}{l}\mathrm{V}=16.99 \mathrm{~m}^{3} \\
\mathrm{~V}_{\mathrm{sd}}=21.24 \mathrm{~m}^{3} \\
\mathrm{D}=2.62 \mathrm{~m} \\
\mathrm{H}=3.93 \mathrm{~m}\end{array}$ \\
\hline
\end{tabular}


Juan B. de León Benítez \& Lesdier Abreu Rodríguez/ITEGAM-JETIA Vol.01, No 04, pp.19-24. Dezembro, 2015.

Tabla 7 - Diseño del condensador de nafta.

Para el diseño del condensador de nafta se utilizó la metodología de cálculo descrita por (Kern D. Q., 1999), para intercambiadores de tubos y concha.

\begin{tabular}{|l|l|l|}
\hline \multicolumn{1}{|c|}{ Datos } & \multicolumn{1}{|c|}{ Fórmulas } & \multicolumn{1}{|c|}{ Resultados } \\
\hline $\mathrm{m}_{\text {nafta }}=2449.58 \mathrm{Kg} / \mathrm{h}$ & $\mathrm{Q}=\mathrm{m}_{\text {nafta }} * \lambda_{\text {nafta }}$ & $\mathrm{Q}=782787.78 \mathrm{KJ} / \mathrm{h}$ \\
$\lambda_{\text {nafta }}=319.56 \mathrm{KJ} / \mathrm{Kg}$ & $\mathrm{MLDT}=\left[\left(\mathrm{T}_{2}-\mathrm{T}_{\mathrm{a} 1}\right)-\left(\mathrm{T}_{1}-\mathrm{T}_{\mathrm{a} 2}\right)\right] / \ln \left[\left(\mathrm{T}_{2}-\mathrm{T}_{\mathrm{a} 1}\right) /\left(\mathrm{T}_{1}-\mathrm{T}_{\mathrm{a} 2}\right)\right]$ & $\mathrm{MLDT}=48.77{ }^{\circ} \mathrm{C}$ \\
$\mathrm{U}_{\mathrm{D}}=1980 \mathrm{KJ} / \mathrm{hm}^{2}{ }^{0} \mathrm{C}$ & $\mathrm{A}=\mathrm{Q} /\left[\mathrm{U}_{\mathrm{D}} * \mathrm{MLDT}\right]$ & $\mathrm{A}=8.11 \mathrm{~m}^{2}$ \\
$\mathrm{~T}_{1}=178^{\circ} \mathrm{C}$ & $\mathrm{m}_{\text {agua }}=\mathrm{Q} /\left[\mathrm{C} \mathrm{p}_{\text {agua }} * \Delta \mathrm{T}_{\text {agua }}\right]$ & $\mathrm{m}_{\text {agua }}=18726.98 \mathrm{Kg} / \mathrm{h}$ \\
$\mathrm{T}_{2}=40^{\circ} \mathrm{C}$ & & \\
$\mathrm{T}_{\mathrm{a} 1}=30^{\circ} \mathrm{C}$ & & \\
$\mathrm{T}_{\mathrm{a} 2}=40^{\circ} \mathrm{C}$ & & \\
$\mathrm{C}_{\text {agua }}=4.18 \mathrm{KJ} / \mathrm{Kg}^{0} \mathrm{C}$ & & \\
\hline
\end{tabular}

\section{RESULTADOS Y DISCUSIÓN}

Como parte de cumplimentar los puntos fundamentales del desarrollo del proceso se dimensionaron los equipos que conforman las etapas de los procesos seleccionados se efectuó el análisis económico de la misma, sobre la base de cálculo del costo de inversión, el costo de la producción, la ganancia y los indicadores de rentabilidad. El estimado de los indicadores se obtuvo aplicándola metodología planteada por Peter's.

Tabla 8 - Elementos del Costo de Inversión.

\begin{tabular}{|c|c|}
\hline Elementos del Costo de Inversión & Costo (\$) \\
\hline Costo de Inversión del Equipamiento & 97970,90 \\
\hline Costos Directos (CD) & 112666.5 \\
\hline Costos Indirectos (CI) & 14496.56 \\
\hline Inversión fija (If) = CD + CI & $\mathbf{1 2 7 1 6 3 , 1}$ \\
\hline Inversión de trabajo (Itr) & $\mathbf{1 4 1 2 9 2 , 3 3}$ \\
\hline Costo Total de Inversión = If + Itr & $\mathbf{2 6 8 4 5 5 . 4 3}$ \\
\hline
\end{tabular}

Tabla 8.1 - Estimado del Costo de Producción.

\begin{tabular}{|c|c|}
\hline Elementos del Costo & Costo (\$/a) \\
\hline Materias Primas & 232377 \\
\hline Costos Variables & 424824 \\
\hline Costos Fijos & 11202 \\
\hline Costos Exteriores & 7212 \\
\hline Costos de Fabricación & $\mathbf{4 4 3 2 3 8}$ \\
\hline Gastos Generales & $\mathbf{4 1 0 2 8}$ \\
\hline Costos Totales de Producción & $\mathbf{4 8 4 2 6 6}$ \\
\hline
\end{tabular}

Tabla 8.3 - Cálculo de la Ganancia.

\begin{tabular}{|l|c|c|}
\hline PRODUCTOS & AAPM & Biodiesel \\
\hline Precio de Venta & $2 \$ / \mathrm{kg}$ & 50 \$/hlt \\
\hline Producción & $9463,9 \mathrm{~kg} / \mathrm{d}$ & $69,0943 \mathrm{hlt} / \mathrm{d}$ \\
\hline $\begin{array}{l}\text { Valor de la } \\
\text { Producción }\end{array}$ & $18927,8 \$ / \mathrm{d}$ & $3454,715 \$ / \mathrm{d}$ \\
\cline { 2 - 4 } $\begin{array}{l}\text { Valor Total de la } \\
\text { Producción }\end{array}$ & $\mathbf{8 1 6 9} \mathbf{6 1 7 , 9 8 5} \$ / \mathbf{a n ̃ o}$ & $\mathbf{6 7 1 4} \mathbf{7 5 4 , 5}$ \$/año \\
\hline
\end{tabular}

$\mathrm{G}=$ Valor Total Producción $(\mathrm{VP})-\mathrm{CTP}$

$G=6230488.5 \$$ / año.

Tabla 8.4 - Valores de los indicadores de factibilidad.

\begin{tabular}{|l|c|}
\hline \multicolumn{1}{|c|}{ Indicador } & Valor \\
\hline Valor Actual Neto (VAN) & $\$ 2279$ \\
& 512,35 \\
\hline Tasa de Rendimiento Interno (TIR) & $56 \%$ \\
\hline Plazo de Recuperación al Descontado (PRD) & 3,4 años \\
\hline
\end{tabular}


Juan B. de León Benítez \& Lesdier Abreu Rodríguez/ITEGAM-JETIA Vol.01, No 04, pp.19-24. Dezembro, 2015.

\section{CONCLUSIONES}

$\checkmark$ En la Empresa Mielera "Heriberto Duquesne" existen las condiciones apropiadas para la implementación de la tecnología de obtención de Biodiesel a partir de un residuo de la industria azucarera (cachaza).

$\checkmark \quad$ La cantidad de cachaza disponible permite obtener una cantidad de Biodiesel de 0,86 ton/d que pudiera ser empleado por la empresa como complemento del combustible para su maquinaria agrícola.

$\checkmark$ En el caso de los balances de energía la planta consumirá mucha energía, por lo que se puede emplear el Biodiesel como combustible para el horno de la fabrica y así disminuir el consumo energético.

$\checkmark$ El análisis técnico - económico es factible gracias a la venta de los Alcoholes de Alto Peso Molecular, por lo que los indicadores reportan un VAN de \$ 2279 512,35; una TIR de $56 \%$ y un PRD de 3,4 años.

\section{REFERENCIAS BIBLIOGRÁFICAS}

[1] Kern, D. Q., (1979). "Procesos de transferencia de calor". La Habana.

[2] Ley Chong, N. "Contribución a los métodos de asimilar tecnologías, aplicado a un caso de producción de Biocombustibles". Tesis en opción al grado científico de Doctor en Ciencias Técnicas. Universidad Central Marta Abreu de las Villas. Julio 2006.

[3] Martínez, R., (2006). "Obtención de biodiesel a partir del aceite de cachaza, residuo de la industria azucarera." Trabajo de Diploma, Santa Clara, Dpto de Ing. Química, UCLV.

\section{[4] Perry, R., (1998). "Chemical Engineers' Handbook." 5ta Edición.}

[5] Rosabal, J., (1998). "Hidrodinámica y separaciones mecánicas”. La Habana.

[6] Treybal, R., (1985). "Operaciones con transferencia de masa." Edición Revolucionaria.

[7] Ulrich, G. "Diseño y Economía de los Procesos de Ingeniería Química".

[8] Villanueva Ramos G. "Memoria Descriptiva: Tecnología para la Obtención de productos de alto valor agregado a partir de la cera cruda de la caña de azúcar". Universidad Central Marta Abreu de las Villas. 2005. 